\title{
Is Laparoscopic Adrenalectomy for Pheochromocytoma a Safe and Effective Surgery in Geriatric Patients?
}

\author{
Kadir Gunseren ${ }^{1}$, Mehmet Çağatay Çiçek ${ }^{1}$, Deniz Bolat ${ }^{2}$, Sezgin Yeni ${ }^{1}$, Hakan Vuruskan ${ }^{1}$, \\ Ozen $\mathrm{Oz} \mathrm{Gul}^{1}$, and Ismet Yavascaoglu ${ }^{1}$ \\ ${ }^{1}$ Uludag University Faculty of Medicine \\ ${ }^{2}$ University of Health Sciences, Izmir Bozyaka Training and Research Hospital
}

March 18, 2021

\begin{abstract}
Objectives: In this study, we aimed to evaluate the haemodynamic changes of laparoscopic adrenalectomy (LA) in geriatric patients with pheochromocytoma (PHEO). To the best of our knowledge, it is the first study evaluating the haemodynamic outcomes of pure LA in this patient population. Methods: Between 2000-2020, data of 350 patients who underwent singleside transperitoneal LA were reviewed retrospectively. Patients with a histopathological diagnosis of PHEO were enrolled and classified into two groups according to their ages at the date of surgery. Older than 65 years were accepted as elderly according to the World Health Organisation recommendations. Results: Overall 54 patients underwent single side LA for PHEO. Fifteen patients were enrolled in the elderly group and 39 in the young group. There were no significant differences regarding the operation site (0.564) and tumour size (0.878). There were no statistically significant differences in terms of perioperative outcomes, such as mean anaesthesia and operation times, blood loss and haemodynamic changes. Regarding postoperative outcomes; there were no statistically significant differences in mean hospitalization and intensive care unit times. In 1 patient in each grade-1 (prolonged ileus, managed with medical treatment) and grade 2 complication (transfusion during surgery) observed in the elderly and young groups, respectively $(\mathrm{p}=0.498)$. Conclusion: Laparoscopic adrenalectomy in elderly patients with PHEO is as safe and effective as in younger patients. One of the most important conditions for achieving good perioperative and postoperative outcomes in the elderly patient population with this challenging surgery is the multidisciplinary approach.
\end{abstract}

\section{Is Laparoscopic Adrenalectomy for Pheochromocytoma a Safe and Effective Surgery in Geri- atric Patients?}

\section{ABSTRACT}

\section{Objectives:}

In this study, we aimed to evaluate the haemodynamic changes of laparoscopic adrenalectomy (LA) in geriatric patients with pheochromocytoma (PHEO). To the best of our knowledge, it is the first study evaluating the haemodynamic outcomes of pure LA in this patient population.

\section{Methods:}

Between 2000-2020, data of 350 patients who underwent single-side transperitoneal LA were reviewed retrospectively. Patients with a histopathological diagnosis of PHEO were enrolled and classified into two groups according to their ages at the date of surgery. Older than 65 years were accepted as elderly according to the World Health Organisation recommendations.

\section{Results:}


Overall 54 patients underwent single side LA for PHEO. Fifteen patients were enrolled in the elderly group and 39 in the young group. There were no significant differences regarding the operation site (0.564) and tumour size (0.878). There were no statistically significant differences in terms of perioperative outcomes, such as mean anaesthesia and operation times, blood loss and haemodynamic changes. Regarding postoperative outcomes; there were no statistically significant differences in mean hospitalization and intensive care unit times. In 1 patient in each grade-1 (prolonged ileus, managed with medical treatment) and grade 2 complication (transfusion during surgery) observed in the elderly and young groups, respectively $(\mathrm{p}=0.498)$.

\section{Conclusion:}

Laparoscopic adrenalectomy in elderly patients with PHEO is as safe and effective as in younger patients. One of the most important conditions for achieving good perioperative and postoperative outcomes in the elderly patient population with this challenging surgery is the multidisciplinary approach.

Keywords: Laparoscopy, Adrenalectomy, Pheochromocytoma, Geriatric, Elderly

\section{INTRODUCTION}

Pheochromocytoma (PHEO) is a neuroendocrine tumour with a prevalence ranging from $0.1-0.6 \%$ in patients with hypertension (1). Paroxysmal hypertension caused by excessive secretion of catecholamines could endanger patients' lives; thus, surgical resection is considered the gold standard treatment for PHEO (1-3). In recent years, it was reported that laparoscopic adrenalectomy (LA) for PHEO is a feasible, safe and superior treatment option (4-6). However, due to the release of catecholamines during operation, surgery for PHEO is still considered as challenging $(4,5)$.

Nowadays, with the increase in life expectancy and advancements in imaging technology, there has been an increase in the diagnosis of PHEO in elderly patients who undergo routine check-ups (7). However, elderly patients are physically more delicate than youngers, and the outcomes of laparoscopic surgery may be considered going to be worse than younger due to hemodynamic instability during operation (8). In this study, we aimed to compare the LA outcomes in elderly and young PHEO patients.

\section{MATERIAL AND METHODS}

After the approval of the institutional ethic committee, we reviewed the database of 350 patients who underwent single side transperitoneal laparoscopic adrenalectomy in our institution between 2000 and 2020 retrospectively. Patients with a confirmed diagnosis of PHEO by histopathological examination were enrolled in the study and were classified into two groups according to their ages at the date of surgery. Older than 65 years were accepted as elderly.

The clinical, hormonal, and radiologic evaluations were performed for all patients. Surgical indications were decided by consulting to the endocrinologist. The premedications of the patients that referred for surgery were administered according to the recommendations of the endocrinology.

Demographic characteristics of patients such as age, sex, body mass index (BMI), American Society of Anesthesiologists Physical Status Classification System (ASA) score, tumour side, tumour size were compared between groups. The size of the tumours were recorded according to the measurement of pathology reports.

Intraoperative and postoperative outcomes, including duration of anaesthesia, operation time, amount of bleeding, hg reduce, haemodynamic measurements during surgery, complications, duration of hospitalization and anti-hypertensive treatment usage ratios were analyzed between groups. Duration of anaesthesia was accepted as the time (minutes) between the induction of anaesthesia and endotracheal extubation of the patient. Operation time was defined as the time (minutes) between incision and skin closure. The amount of bleeding (ml) was determined by measuring the amount of fluid in the aspirator after surgery. Haemoglobin reduce was determined by measuring the difference in haemoglobin levels between the preoperative and postoperative first days laboratory results.

In this study, we aimed to evaluate hemodynamic instability more accurately, and due to the possibility of the 
hypertensive episode during endotracheal intubation in PHEO patients, we analyzed the tension and pulse records in the duration of anaesthesia instead of the duration of the operation (9). Additionally, we recorded and analyzed different hemodynamic parameters, including inlet systolic blood pressure (SBP), inlet diastolic blood pressure (DBP), maximum and minimum SBP and DBP and also the systolic and diastolic instabilities. The numbers of episodes of SBP $>200 \mathrm{mmHg}$, SBP $>160 \mathrm{mmHg}$, mean arterial pressure (MAP) $<60 \mathrm{mmHg}$, SBP above $30 \%$ the baseline, DBP below $30 \%$ the baseline and heart rate $(\mathrm{HR})>110$ were evaluated. Inlet systolic and diastolic pressures $(\mathrm{mmHg})$ were described as the measurement of the blood pressure before the intubation and administration of the surgical position. Systolic and diastolic instabilities were defined as the differences between maximum and minimum systolic and diastolic blood pressures $(\mathrm{mmHg})$.

Complications were classified according to the Clavien Dindo scoring system and compared between groups. The ratios of using and leaving the anti-hypertensive (HT) treatments in the groups were evaluated.

\section{Surgical Technique}

All operations were performed using the standard transperitoneal lateral laparoscopic approach. The patients were placed right lateral decubitis position for left LA, while they were placed right lateral decubitis position for left LA. Pneumoperitoneum was established using a Veress needle technique, and four trocars were used for both right and left LA. The intraperitoneal pressure was adjusted to $12 \mathrm{mmHg}$ during the surgery.

\section{Left Adrenalectomy:}

The Told line was incised from splenic flexure to the sigmoid junction to mobilization the left colon. After reflecting the descending colon medially, the dissection was carried out between Gerota's fascia and the mesocolon up to access to the renal and adrenal vein. Dissection of the adrenal gland before vascular control was avoided to prevent the systemic release of catecholamines. The adrenal vein was controlled using Hemo-Lock clips, and dissection was continued from just superior to the renal vein until the psoas muscle was identified. The tissue was manipulated without directly grasping the adrenal gland to avoid bleeding and potential hemodynamic instability. The medial dissection was carried out from the renal vein to the crus of the diaphragm. An ultrasonic scalpel was used to control small vessels. Once the medial dissection was completed, lateral dissection was extended between the renal cortex and perinephric fat adjacent to the adrenal gland. The specimen was removed using an endopouch.

\section{Right Adrenalectomy:}

As the first step, the liver was retracted, and subhepatic inferior vena cava was identified by mobilization of colon or duodenum if required. The peritoneum was incised to expose the lateral edge of vena cava and the superior edge of the renal vein. The dissection was extended from the superior border of the renal vein until the psoas muscle was exposed. The dissection was continued just lateral of the vena cava until the right adrenal vein was identified. Manipulating the adrenal gland was avoided to prevent the systemic release of catecholamines. The adrenal vein was controlled using Hem-o-Lock clips, and the dissection was continued to superiorly below the liver until the lateral abdominal wall was visualized. Once the medial dissection was completed, the lateral dissection was performed just as described for left adrenalectomy. The specimen was removed using an endopouch.

\section{Statistical Analyses:}

Data were analyzed using SPSS v 22 software program for Windows (SPSS, Inc., Chicago, IL USA). Distributions of the values were evaluated with the Kolmogorov-Smirnov Test. While the Independent Sample Student t-test was used for the analysis of variables with normal distribution, the variables without normal distribution were evaluated by using the Mann-Whitney U test. Data were presented as mean \pm SD or median (range) according to the distribution of the values. Chi-squared or Fisher tests were used to compare categorized variables. Values were considered significant if the p-value was $<0.05$.

\section{RESULTS}


Fifty-four patients who underwent single side laparoscopic adrenalectomy in our clinic were included in the study. Fifteen patients were enrolled in the elderly group and 39 in the young group according to their ages at the date of surgery. The demographic characteristics of the groups were summarised in Table 1 . The mean ages of the two groups were $66.8 \pm 2.5$ years and $41.2 \pm 10$ years $(\mathrm{p}=0.000)$. While male patients ratios were $73.3 \%$ and $41 \%$ in elderly and young groups, respectively, the female patients' ratio were $26.7 \%$ and $50 \%$ $(\mathrm{p}=0.033)$. No difference was observed for BMI between the groups, whereas the ASA score was higher in the elderly group $(\mathrm{p}=0.023)$. The ratio of the operation sides was similar between groups $(\mathrm{p}=0.565)$. The mean pathologic size was $48.07 \pm 12.6 \mathrm{~mm}$ vs $47.24 \pm 19 \mathrm{~mm}$ in elder and young groups, respectively ( $\mathrm{p}=0.878$ ).

Intraoperative outcomes of the groups were presented in Table 2. While the mean duration of anaesthesia was $161 \pm 42.6 \mathrm{~min}$ and $152.5 \pm 44.9 \mathrm{~min}$ in elderly and young groups $(\mathrm{p}=0.278)$, respectively, the mean operation time was $113.3 \pm 39.4 \mathrm{~min}$ vs $108.38 \pm 39.8 \mathrm{~min}(\mathrm{p}=0.685)$. The amount of bleeding and hg reduce ratios were similar between groups $(\mathrm{p}=0.958$ and $\mathrm{p}=0.472$, respectively) (Table 2$)$. Additionally, the various haemodynamic parameters of the patients under the anaesthesia were similar between elder and young groups, as presented in Table 2 .

Postoperative outcomes of the groups were presented in Table 3. None of the patients in the groups stayed in the intensive care unit postoperatively. There was no difference in terms of the duration of hospitalization and complication ratio between groups $(\mathrm{p}=0.156$ and $\mathrm{p}=0.498$, respectively) (Table 3$)$. According to the Clavien-Dindo classification, among the perioperative and postoperative period, there was one grade 1 complication (prolonged ileus, managed with medical treatment) in the elder group and one grade 2 complication (transfusion during surgery) in the young group. There was no conversion to open surgery in groups. The proportion of leaving anti-hypertensive therapy was $46.7 \%$ vs $59 \%$ in elderly and young groups, respectively $(\mathrm{p}=0.415)$ (Table 3).

\section{DISCUSSION}

The elderly population (i.e., the group of patients aged 65 years or older) is growing all over the world. Demographic estimates show that the human population aged 65 years or older will exceed 1.5 billion in 2050 (10). Increasing life expectancies and improvements in medical technology and healthcare lead to an increase in the number of elder patients undergoing complex surgical procedures (11). Elderly patients have several physiologic changes such as cardiovascular, pulmonary and hepatorenal that impact their response to anaesthesia, surgical stress and postoperative rehabilitation (12). In this context, laparoscopic approaches with benefits, including less postoperative pain or more rapid return to regular daily activities are well suited to the challenges of elderly individuals (13). On the other hand, the increased physiologic demands of laparoscopic approaches may present particular challenges on elderly patients (11). This situation may be more critical, especially in surgeries that hemodynamic parameters are affected, such as PHEO.

Pheochromocytoma is a tumour that originates from adrenomedullary chromaffin cells and releases one or more of the following catecholamines: epinephrine, norepinephrine and dopamine (14). The most clinical characteristics such as sweating, headache, palpitations and episodic hypertension are secondary to the secretion of these catecholamines (14). Surgical management is considered the gold standard treatment of PHEO (1-3). Although the laparoscopic resection of PHEO was reported as feasible and safe, there is a conviction as the fluctuation of catecholamine levels during operation makes this surgery as challenging (15). The incidence of hemodynamic instability during resection of PHEO ranges between $17 \%$ to $83 \%$ (16). Studies that specifically investigated this topic is limited in the literature. Thus, whether the hemodynamic changes during laparoscopic treatment of PHEO constitutes a more surgical risk for elderly patients than youngers and the comparison of the outcomes according to the ages deserves additional study.

There is no standard definition of intraoperative hemodynamic instability in the literature (17). In this study, we defined hemodynamic instability as the difference between max and min systolic and diastolic blood pressures. Additionally, we compared the ratio of SBP $>200 \mathrm{mmHg}, \mathrm{MAP}<60 \mathrm{mmHg}, \mathrm{HR}>110$ and the ratio of $30 \%$ above and below baseline measurements of blood pressures between two groups to evaluate hemodynamic changes more accurately. According to our findings, there was no difference between elder 
and young patients in terms of hemodynamic instability during laparoscopic PHEO surgery. Bruynzeel et al. reported that age was not associated with hemodynamic instability during surgery of PHEO; however, in this study, laparoscopic and open surgeries were included and patients were not stratified by age (18). Similarly, Srougi et al. reported that being older than 60 years did not affect hemodynamic instability; however, their study included open and laparoscopic adrenalectomies and also partial and bilateral adrenalectomies (8). In this context, our study differs from previous reports by analyzing the pure laparoscopic and complete resections between elder and young patients.

The length of hospitalization of elderly patients that undergo surgery may be expected as to be longer due to physiological changes of ageing. Colins et al. reported that advanced age is a predicting factor for length of hospital stay; however, intraoperative and postoperative factors are more predictive than age (19). This may be also valid for elderly patients that undergo a challenging surgery such as PHEO. In our study, we found that the length of hospital stay was similar between elderly and young patients. We think that being no difference between the groups in terms of hemodynamic instability and complication rates are the most important reason for our these finding. Because, most simply, even the elevated blood pressure due to catecholamines may cause haemorrhage either intra or postoperatively (20). This is a major complication that may affect the duration of hospitalization. The postoperative period is usually smoothly in intraoperatively uncomplicated, calmly cases. Our results indicate that laparoscopic PHEO surgery has successful results in the elderly, just like in young individuals, in the absence of HI.

The similarity of complication rates and length of hospital stay between elderly and younger individuals in our study also reveals the importance of premedication and anaesthetic management indirectly. The mortality after surgery of PHEO can reach up to almost $50 \%$ in unprepared patients (21). Therefore, the pre-requisite of the obtain successful outcomes in PHEO surgery is the close co-ordination and co-operation between anesthesiologist, surgeon and endocrinologist (21). The multidisciplinary approach provides faster recovery with no or minimal complications in the postoperative period (20). According to our institution's management of PHEO patients, all individuals are evaluated by the collaboration of endocrinologist, anesthesiologist and a urologist. Indeed, in the well pre-medicated cases, intraoperative problems such as hemodynamic stabilities or bleeding were not usual, even in elderly patients as presented in our results. Consequently, it can be said that well premedication has a positive feedback effect; it reduces the hemodynamic instability and risk of complications such as bleeding, and this decrement reduces the stress on the surgeons' shoulders and more reduces the risk of complications by leading him/her to do the job more meticulously.

Additionally, our findings demonstrate that the ratio of leaving anti-HT treatment in the postoperative period is found as similar between elderly and young patients. According to our results, LA in the management of PHEO gives effective results in elderly patients as well as young individuals, in terms of HT control in the postoperative period.

Although our study is the first which comparing the surgical outcomes of pure LA between elderly and young patients with PHEO in the literature, it has some limitations. First, it is a retrospective designed study. Second, although the conduction of the present study in the single centre is an advantage, on the other hand as a natural result of PHEO incidence being as 3 to 8 per 1 million adults, it has a small sample size (14). Third, we performed all the LAs with a transperitoneal approach, so we don't have the data of the retroperitoneal approach.

\section{CONCLUSIONS}

Laparoscopic adrenalectomy in elderly patients with PHEO is as safe and effective as in younger individuals. One of the most important conditions for achieving good perioperative and postoperative outcomes in the elderly patient population with this challenging surgery is the multidisciplinary approach. Accurate preoperative diagnosis, preparation and also with proper anaesthetic and surgical management, LA could become safe and effective procedure in elderly patients with PHEO.

\section{ACKNOWLEDGMENTS}


None

\section{CONFLICTS OF INTEREST}

The authors declare that they have no conflict of interest.

\section{FUNDING}

None

\section{REFERENCES:}

1. Lenders JW, Eisenhofer G, Mannelli M, Pacak K: Phaeochromocytoma. Lancet 2005; 366(9486):665-75.

2. Schürmeyer TH, Engeroff B, Dralle H, von zur Mühlen A: Cardiological effects of catecholamine-secreting tumours. Eur J Clin Invest. 1997; 27(3):189-95.

3. Zeiger MA, Thompson GB, Duh QY, Hamrahian AH, Angelos P. Elaraj D et al. American Association of Clinical Endocrinologists and American Association of Endocrine Surgeons Medical Guidelines for the Management of Adrenal Incidentalomas: executive summary of recommendations. Endocr Pract 2009 ;15(5):450.

4. Li J, Wang Y, Chang X, Han Z: Laparoscopic adrenalectomy (LA) vs open adrenalectomy (OA) for pheochromocytoma (PHEO): A systematic review and meta-analysis. Eur J Surg Oncol. 2020; 46(6):991-8.

5. Zhu W, Wang S, Du G, Liu H, Lu J, Yang W: Comparison of retroperitoneal laparoscopic versus open adrenalectomy for large pheochromocytoma: a single-center retrospective study. World J Surg Oncol. 2019; 17(1):111.

6. Bai S, Yao Z, Zhu X, Li Z, Jiang Y, Wang R: Comparison of transperitoneal laparoscopic versus open adrenalectomy for large pheochromocytoma: A retrospective propensity score-matched cohort study. Int J Surg. 2019; 61:26-32.

7. Kalache A, Keller I: Population ageing in developing countries: demographic aspects. In: Evans JG, Williams TF, Beattie BL, Michel JP, Wilcock GK. Oxford Textbook of Geriatric Medicine. New York: Oxford University Press. 2000; pp. 26-32.

8. Srougi V, Chambo JL, Tanno FY, Soares IS, Almeida MQ, Pereira MAAet al. Presentation and surgery outcomes in elderly with pheocromocytoma: a comparative analysis with Young patients. Int Braz J Urol. 2016; 42(4):671-7.

9. Vorselaars WMCM, Postma EL, Mirallie E, Thiery J, Lustgarten M, Pasternak JD et al. Hemodynamic instability during surgery for pheochromocytoma: comparing the transperitoneal and retroperitoneal approach in a multicenter analysis of 341 patients. Surgery. 2018; 163(1):176-82.

10. United Nations, Department of Economic and Social Affairs, Population Division. World Population Ageing 2019: Highlights. New York. digitallibrary.un.org. [internet]. Available from: https://digitallibrary.un.org/record $/ 3846855 ? \mathrm{ln}=\mathrm{en}$.

11. Caglià P, Tracia A, Buffone A, Amodeo L, Tracia L, Amodeo C et al. Physiopathology and clinical considerations of laparoscopic surgery in the elderly. Int J Surg. 2016; 33(1):97-102

12. Yang R, Wolfson M, Lewis MC: Unique Aspects of the Elderly Surgical Population: An Anesthesiologist's Perspective. Geriatr Orthop Surg Rehabil 2011; 2(2):56-64.

13. Bates AT, Divino C: Laparoscopic surgery in the elderly: a review of the literature. Aging Dis. 2015; 10(2):149-55.

14. Sbardella E, Grossman AB: Pheochromocytoma: An approach to diagnosis. Best Pract Res Clin Endocrinol Metab. 2020; 34(2):101346. 
15. Conzo G, Musella M, Corcione F, De Palma M, Avenia N, Milone M et al. Laparoscopic treatment of pheochromocytomas smaller or larger than $6 \mathrm{~cm}$. A clinical retrospective study on 44 patients. Laparoscopic adrenalectomy for pheochromocytoma. Ann Ital Chir. 2013; 84(4):417-22.

16. Scholten A, Vriens MR, Cromheecke GJ, Borel Rinkes IH, Valk GD: Hemodynamic instability during resection of pheochromocytoma in MEN versus non-MEN patients. Eur J Endocrinol. 2011; 165(1):91-6.

17. Ma L, Shen L, Zhang X, Huang Y: Predictors of hemodynamic instability in patients with pheochromocytoma and paraganglioma. J Surg Oncol. 2020; 122(4):803-8.

18. Bruynzeel H, Feelders RA, Groenland THN, van der Meiracker AH, van Eijck CHJ, Lange JF et al. Risk Factors for Hemodynamic Instability during Surgery for Pheochromocytoma. J Clin Endocrinol Metab. 2010; 95(2):678-85.

19. Collins TC, Daley J, Henderson WH, Khuri SF: Risk factors for prolonged length of stay after major elective surgery. Ann Surg. 1999; 230(2):251-259.

20. Mamilla D, Araque KA, Brofferio A, Gonzales MK, Sullivan JN, Nilubol N et al. Postoperative Management in Patients with Pheochromocytoma and Paraganglioma. Cancers (Basel). 2019; 11(7):936.

21. Bajwa SS, Bajwa SK: Implications and considerations during pheochromocytoma resection: A challenge to the anesthesiologist. Indian J Endocrinol Metab. 2011; 15(4):337-344.

\section{FIGURE LEGENDS:}

Table 1: Demographic characteristics of the patients with pheochromocytoma

Table 2: Intraoperative parameters and hemodynamics of the patients

Table 3: Postoperative outcomes and medical antihypertensive treatment ratios.

\section{Hosted file}

int $j$ clin pract, pheo elderly, table 1.pdf available at https://authorea.com/users/366204/ articles/514164-is-laparoscopic-adrenalectomy-for-pheochromocytoma-a-safe-and-effectivesurgery-in-geriatric-patients

\section{Hosted file}

int $j$ clin pract, pheo elderly, table 2.pdf available at https://authorea.com/users/366204/ articles/514164-is-laparoscopic-adrenalectomy-for-pheochromocytoma-a-safe-and-effectivesurgery-in-geriatric-patients

\section{Hosted file}

int $j$ clin pract, pheo elderly, table 3.pdf available at https://authorea.com/users/366204/ articles/514164-is-laparoscopic-adrenalectomy-for-pheochromocytoma-a-safe-and-effectivesurgery-in-geriatric-patients 